\title{
Zero-field $J$-Spectroscopy of Urea: Spin-Topology Engineering by Chemical-Exchange
}

\author{
Seyma Alcicek, ${ }^{1, *}$ Piotr Put, ${ }^{1}$ Danila Barskiy,${ }^{2,3}$ Vladimir Kontul, ${ }^{1}$ and Szymon Pustelny ${ }^{1, \dagger}$ \\ ${ }^{1}$ Institute of Physics, Faculty of Physics, Astronomy and Applied Computer Science, \\ Jagiellonian University in Kraków, 30-348 Kraków, Poland \\ ${ }^{2}$ Helmholtz Institute Mainz, GSI Helmholtz Center for Heavy Ion Research GmbH, 55128 Mainz, Germany \\ ${ }^{3}$ Institute of Physics, Johannes Gutenberg-Universität, 55128 Mainz, Germany
}

\begin{abstract}
Well-resolved and information-rich $J$-spectra are the foundation for chemical analysis based on zero-field NMR. Yet, even in relatively small molecules, the spectra may gain complexity, hindering the analysis. To address this problem, we investigate an example biomolecule characterized with a complex $J$-coupling network - urea, a key metabolite in protein catabolism - and demonstrate ways of simplifying its zero-field spectra by modifying spin topology. This goal is achieved by controlling $\mathrm{pH}$-dependent chemical-exchange rates of ${ }^{1} \mathrm{H}$ nuclei and varying the composition of the $\mathrm{D}_{2} \mathrm{O} / \mathrm{H}_{2} \mathrm{O}$ mixture used as a solvent. Specifically, we demonstrate that by increasing hydrogen chemicalexchange rate in $\left[{ }^{13} \mathrm{C},{ }^{15} \mathrm{~N}_{2}\right]$-urea solution, the molecule, being an effective spin system $\mathrm{XAB}_{2} \mathrm{~A}^{\prime} \mathrm{B}^{\prime}{ }_{2}$, behaves as a much simpler $\mathrm{XA}_{2}$ system (where $\mathrm{X}={ }^{13} \mathrm{C}, \mathrm{A}={ }^{15} \mathrm{~N}, \mathrm{~B}={ }^{1} \mathrm{H}$ ), manifesting through a single narrow spectral peak. Additionally, we show that introducing spin-1 nuclei into the molecule and investigating $J$-spectra of ${ }^{1} \mathrm{H} / \mathrm{D}$ isotopologues of $\left[{ }^{15} \mathrm{~N}_{2}\right]$-urea allows to study various isolated spin subsystems: $\mathrm{XA}_{2},(\mathrm{XA}) \mathrm{B}$, and $\mathrm{XB}_{2}$ (here $\left.\mathrm{X}={ }^{15} \mathrm{~N}, \mathrm{~A}={ }^{1} \mathrm{H}, \mathrm{B}=\mathrm{D}\right)$, again greatly simplifying spectra analysis. The influence of the chemical exchange process on zero-field $J$-spectra for each urea solution is elucidated by theoretical studies, demonstrating solid agreement between results and simulations. This study shows the applicability of zero-field NMR to detect complex biomolecules in aqueous solutions, and it opens the means for future in vivo/in vitro biochemical investigations, particularly in biofluids with a high concentration of water.
\end{abstract}

\section{INTRODUCTION}

Zero- and ultralow-field (ZULF) nuclear magnetic resonance (NMR) is a novel, portable, and cost-effective technique that enables high-precision chemical analysis through direct observation of intramolecular spin interactions at truly zero or very weak (weaker than $100 \mathrm{nT}$ ) external magnetic fields [1-6]. Since in isotropic liquids direct magnetic dipolar interaction averages out to zero, in molecules with nuclei of spin smaller than one (absence of quadrupolar interaction), under the zero-field regime, an indirect spin-spin coupling (also known as $J$ coupling) becomes dominant interaction [7]. This allows to use zero-field NMR for the determination of a whole network of $J$-couplings in the molecule and hence enables identification and quantification of (bio-)chemical compounds [8,9]. Furthermore, since exchange processes alter spin-spin couplings, this approach also enables monitoring chemical-exchange processes, involving, for example, chemical reactions (bond-breaking, bondmaking) or conformational modifications (bond rotation) [10]. Thereby, ZULF NMR arises as an appealing spectroscopic modality for studies of chemical exchange of small biomolecules [11]. This application was recently demonstrated in a context of molecules consisting of 25 coupled nuclear spins $[6,12]$, but, for large spin systems, ZULF NMR spectra becomes complicated, due to increasing number of coupled nuclei, and its analysis is challenging. In this scope, herein, we present various ap-

\footnotetext{
* seyma.alcicek@uj.edu.pl

† szymon.pustelny@uj.edu.pl
}

proaches of modifying and simplifying zero-field spectra of molecules, containing a large number of spins some of which can undergo a chemical exchange. For this purpose, we implement ZULF NMR $J$-spectroscopy to investigate aqueous solutions of urea, a molecule with a large spin coupling network and prominent proton exchange.

Urea plays a vital role in amino-acid and protein metabolism, enabling 80-90\% of nitrogen excretion from the human body. It is generated in the liver in the urea cycle, transported via the bloodstream, and excreted into the urine by the kidneys [13]. For this reason, measuring urea level in urine and/or blood is routinely used medical diagnostic technique to evaluate liver and kidney function [14-16]. Moreover, $\left[{ }^{13} \mathrm{C}\right]$-urea and $\left[{ }^{13} \mathrm{C},{ }^{15} \mathrm{~N}_{2}\right]$-urea have recently become attractive contrast agents for hyperpolarized magnetic resonance imaging studies, making the compounds a valuable marker for the evaluation of myocardial perfusion and renal physiology [17-19]. Finally, the interest in urea is also stimulated by a growing demand for robust and reliable instrumentation for compound detection in such fields as environmental monitoring as well as agricultural and food chemistry [20].

In our work, we investigate solutions of $\left[{ }^{15} \mathrm{~N}_{2}\right]$ - and $\left[{ }^{13} \mathrm{C},{ }^{15} \mathrm{~N}_{2}\right]$-urea in various solution environments by observing changes in the compounds' zero-field NMR $J$ spectra. First, we demonstrate the influence of a protonexchange process on $J$-spectra by measuring $\left[{ }^{15} \mathrm{~N}_{2}\right]$-urea and $\left[{ }^{13} \mathrm{C},{ }^{15} \mathrm{~N}_{2}\right]$-urea in an aprotic solvent, dimethyl sulfoxide (DMSO), and a protic solvent, water $\left(\mathrm{H}_{2} \mathrm{O}\right)$. Since a proton-exchange rate in urea is both acid- and basecatalyzed, we also investigate aqueous solutions of $\left[{ }^{15} \mathrm{~N}_{2}\right]$ urea and $\left[{ }^{13} \mathrm{C},{ }^{15} \mathrm{~N}_{2}\right]$-urea at various $\mathrm{pH}$ levels. The results are elucidated by zero-field NMR simulations of 
chemical exchange and nuclear spin dynamics using a simple theoretical model. $\left[{ }^{15} \mathrm{~N}_{2}\right]$-urea is also measured in mixtures of $\mathrm{H}_{2} \mathrm{O}$ and $\mathrm{D}_{2} \mathrm{O}$ to study the effect of $\mathrm{D}$ nuclei on the zero-field $J$-spectra. The experimental results are supported by simulations taking into account the proportion of ${ }^{1} \mathrm{H} / \mathrm{D}$ isotopologues of urea in solution. All spectral peaks, arising from $J$-coupling interactions $\left({ }^{15} \mathrm{~N}-{ }^{1} \mathrm{H},{ }^{15} \mathrm{~N}-\mathrm{D}\right.$, and $\left.{ }^{1} \mathrm{H}-\mathrm{D}\right)$ in spin subsystems, are assigned by analyzing the energy-level structure of isotopologues using perturbation theory. Based on the presented results, we show straightforward ways to study complex biomolecules in ZULF NMR by taking advantage of the chemical-exchange process.

\section{RESULTS AND DISCUSSION}

\section{A. Zero-field $J$-spectra of urea in protic and aprotic solvents}

Urea molecule contains two $-\mathrm{NH}_{2}$ groups joined by a carbonyl $(\mathrm{C}=\mathrm{O})$ functional group. To analyse the general structure of $J$-spectra of $\left[{ }^{15} \mathrm{~N}\right]$-urea and $\left[{ }^{13} \mathrm{C},{ }^{15} \mathrm{~N}_{2}\right]$ urea, first, numerical simulations are performed using $J$-coupling constants shown in Fig. $1[21,22]$. Since one-bond ${ }^{1} \mathrm{H}^{15} \mathrm{~N}$ coupling in the $-\mathrm{NH}_{2}$ group is the strongest interaction in this system, the main features in the $J$-spectra of both forms of urea are centered around $(3 / 2)\left|{ }^{1} J_{\mathrm{NH}}\right| \approx 133.65 \mathrm{~Hz}$ (marked by a dashed line in Fig. 1), as expected for the $\mathrm{XA}_{2}$ nuclear spin system corresponding to the transitions in the manifold with the total proton spin 1 , see for example [8, 23]. Hereafter, we refer to this group of signals as high-frequency peaks. Other (weaker) homonuclear and heteronuclear interactions result in the appearance of so-called lowfrequency peaks $(<10 \mathrm{~Hz})$, as well as, further modifications (splitting and shifting) of the main high-frequency peaks. Specifically, the presence of an additional, relatively strong ${ }^{13} \mathrm{C}-{ }^{15} \mathrm{~N}$ interaction in $\left[{ }^{13} \mathrm{C},{ }^{15} \mathrm{~N}_{2}\right]$-urea increases the shifts in corresponding energy manifolds, giving rise to a wider span of low- and high-frequency peaks compared to the spectrum of $\left[{ }^{15} \mathrm{~N}_{2}\right]$-urea.

Figure 1 compares $J$-spectra of both forms of urea in dimethylsulfoxide (DMSO) and water. The experimental spectrum in DMSO agrees well with the simulated spectrum, especially in terms of peak positions. In contrast, lines become substantially broader ( $\sim 3$ times) when water is used as a solvent (Fig. 1). This is expected since amide protons are known to undergo chemical exchange with water protons and this process contributes to nuclear spin relaxation rate [24]. However, the mere fact of being able to observe these multiplets in $J$-spectra indicates that the proton chemical-exchange rate in urea at neutral $\mathrm{pH}$ level is slow enough for ZULF NMR measurements compared to the spin evolution due to $J$-couplings. Also, this is confirmed by a chemical-exchange rate for neutral $\mathrm{pH}$ being equal to approximately $1.9 \mathrm{~s}^{-1}$, which is significantly slower than dominant interaction in the

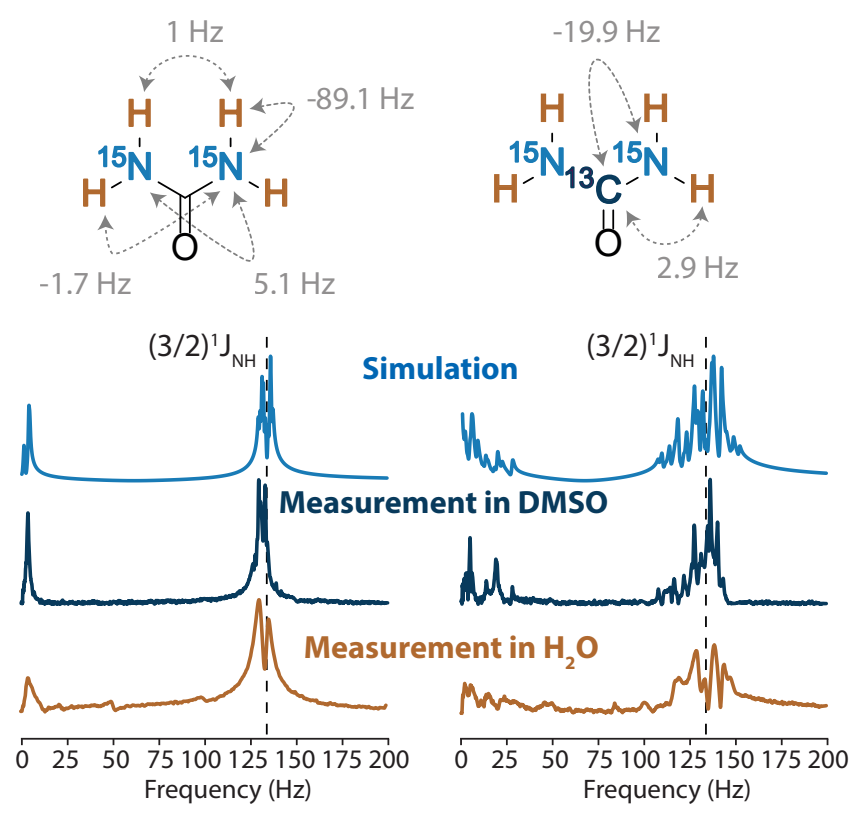

FIG. 1. Simulated and experimental ZULF NMR spectra of $\left[{ }^{15} \mathrm{~N}_{2}\right]$-urea and $\left[{ }^{13} \mathrm{C},{ }^{15} \mathrm{~N}_{2}\right]$-urea in aprotic (DMSO) and protic $\left(\mathrm{H}_{2} \mathrm{O}\right)$ solvents. The $J$-coupling values used in the simulation are shown with structural formulas.

system $\left({ }^{1} J_{\mathrm{NH}}\right)[24]$.

It is worth noting that, due to the absence of heteronuclear spin-spin coupling, water does not give rise to ZULF NMR peaks at a truly zero magnetic field. This can be seen as an advantage of zero-field NMR compared to high-field ${ }^{1} \mathrm{H}$ NMR, where water protons give rise to a very intense and broad signal that can overlap with other signals [25]. The feature makes zero-field NMR a promising modality for the analysis of biological samples with a high concentration of water (e.g., blood, urine, or cell cultures) as there is no need for solvent suppression.

\section{B. Effects of pH-dependent chemical exchange on zero-field spectra}

Proton-exchange process in aqueous solutions of urea is pH-depended and it is both acid- and base-catalysed. Thus, herein, we distinguish two exchange processes [2629]:

$$
\begin{gathered}
\mathrm{CON}_{2} \mathrm{H}_{4}+\mathrm{H}^{+} \rightleftharpoons \mathrm{CON}_{2} \mathrm{H}_{5}^{+} \\
\mathrm{CON}_{2} \mathrm{H}_{4}+\mathrm{OH}^{-} \rightleftharpoons \mathrm{CON}_{2} \mathrm{H}_{3}^{-}+\mathrm{H}_{2} \mathrm{O}
\end{gathered}
$$

In this work, the effect of proton-exchange rate on $J$ spectra is studied by varying $\mathrm{pH}$ of the solution while keeping the same concentration of urea $(8 \mathrm{M})$. As shown in Fig. 2, due to the increased proton-exchange rate in urea solutions, amplitudes of high-frequency peaks (120$150 \mathrm{~Hz}$ ) gradually decrease without considerable line 
broadening at both lower and higher $\mathrm{pH}$ values. It is clear that, when the proton-exchange rate is much higher than $J$-coupling interaction $\left(k_{\mathrm{ex}} \gg J_{\mathrm{NH}}\right),{ }^{1} \mathrm{H}$ nuclei is effectively decoupled from the rest of the spin system and the $J_{\mathrm{NH}}$-coupling does not contribute to the observed zerofield spectra. Therefore, high-frequency peaks vanish in the spectra of highly acidic $(\mathrm{pH}=1.4)$ and highly basic $(\mathrm{pH}=14)$ solutions, which is also confirmed with simulations of the urea spin system (Fig. 2, see also Methods). The disappearance of the high-frequency peaks in highly acidic/basic conditions also bears a resemblance to the results shown in a recent study on zero-field NMR of ammonium in highly acidic conditions [11]. The authors of Ref. [11] reported that an increase in the proton-exchange rates causes the zero-field NMR signals of ammonium to vanish. This is explained by the nature of the experiment: after prepolarization in a strong field, a sample spends a significant amount of time (1s) in a low-field region (tens of $\mu \mathrm{T}$ ) before the detection in zero-field. In our experiment, we are limited to a shuttling time (time between prepolarization and signal acquisition) of $1 \mathrm{~s}$, as for the shorter transfer times vibration noise, stemming from a transport of the NMR tubes, disrupts the structure of the spectra. For such a delay, water protons depolarize despite a guiding field of $10 \mu \mathrm{T}$. In the case of faster proton exchange, unpolarized protons are more often involved in the exchange procedure. This affects the "memory" of nuclei spin orders, resulting in the deterioration of amplitudes of peaks. To verify the influence of guiding field strength on the peak amplitude, the field was increased by an order of magnitude, which, due to the increased proton relaxation time $T_{1}$, resulted in an up to $\approx 25 \%$ signal enhancement (Fig. S1) [30]. The increase of the signal amplitude in a higher guiding field is predicted to be universal for molecules under rapid chemical exchange and can be exploited in measuring zero-field spectra of such molecules. The boost in the signal stemming from a stronger "transfer" field may not only be beneficial for remote prepolarization experiments but also find use in ZULF hyperpolarization techniques relying on the chemical exchange [31, 32], where a stronger magnetic field will slow down the relaxation of protons in solution and yield higher signal amplitudes in subsequent zero-field detection.

On the other hand, rapid proton exchange leads to a modification of the effective coupled spin system that can greatly simplify the spectra. This is demonstrated in the spectra of $\left[{ }^{13} \mathrm{C},{ }^{15} \mathrm{~N}_{2}\right]$-urea in highly acidic and basic solutions (red boxes in Fig. 2), where a narrow peak appears at $\approx 30 \mathrm{~Hz}$. This signal arises at $(3 / 2) J_{\mathrm{CN}}$ and originates from the $J$-coupling between ${ }^{13} \mathrm{C}$ and ${ }^{15} \mathrm{~N}$ nuclei in a $\mathrm{CN}_{2}$ spin system, where, due to the rapid exchange, protons are effectively decoupled from the rest of the nuclei. The emergence of the low-frequency peak is also supported by the numerical simulations for the spin system under rapid chemical exchange (shown in the bottom of Fig. 2). It should be stressed that, by taking advantage of the accelerated chemical-exchange effect on the zero-field NMR, a

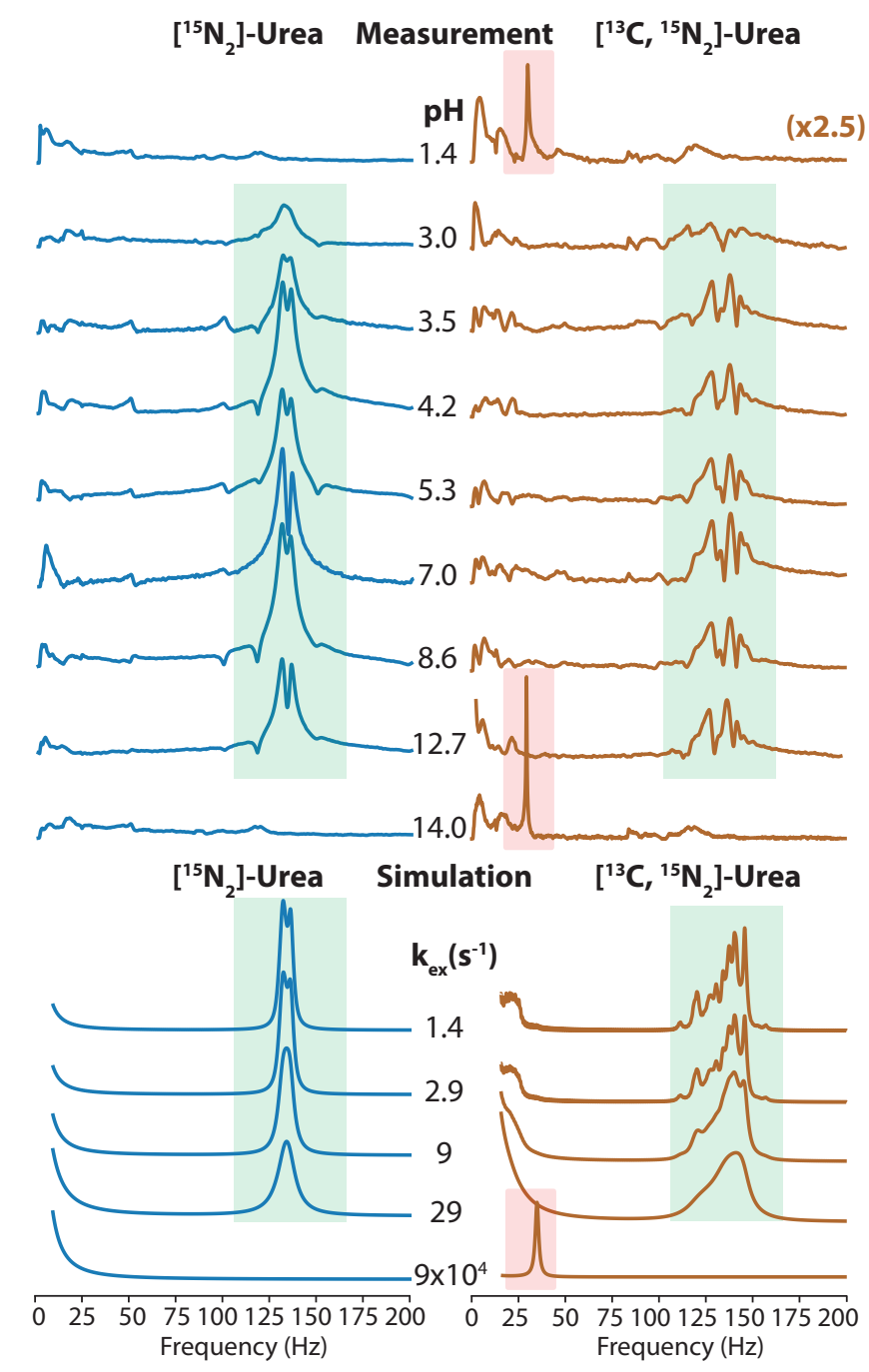

FIG. 2. Experimental (top) and simulated (bottom) zerofield $J$-spectra of $\left[{ }^{15} \mathrm{~N}_{2}\right]$-urea and $\left[{ }^{13} \mathrm{C},{ }^{15} \mathrm{~N}_{2}\right]$-urea in aqueous solutions at various $\mathrm{pH}$ values. The peaks arising from onebond strong $J$-coupling interaction between ${ }^{15} \mathrm{~N}$ and ${ }^{1} \mathrm{H}$ are green shaded $(120-150 \mathrm{~Hz})$, while the narrow peaks (around $30 \mathrm{~Hz}$ ) originating from one-bond $J$-coupling between ${ }^{13} \mathrm{C}$ and ${ }^{15} \mathrm{~N}$, are highlighted in red.

narrower single peak (1-Hz width) with higher amplitude was obtained owing to modification of the spin topology from the complex $\mathrm{XAB}_{2} \mathrm{~A}^{\prime} \mathrm{B}_{2}$ ' system to the simple $\mathrm{XA}_{2}$ system.

\section{Zero-field $J$-spectra of deuterium isotopologues of urea}

Next, we investigated ${ }^{1} \mathrm{H} / \mathrm{D}$ isotopologues of urea. For these studies, we modify the spin coupling network by replacing ${ }^{1} \mathrm{H}$ (spin-1/2) with $\mathrm{D}$ (spin-1), by solving urea in $\mathrm{D}_{2} \mathrm{O} / \mathrm{H}_{2} \mathrm{O}$ mixture. To simulate $J$-spectra of urea solutions with various $\mathrm{D}_{2} \mathrm{O} / \mathrm{H}_{2} \mathrm{O}$ ratios, the proportion of each isotopologue in a solution is calculated using a bino- 


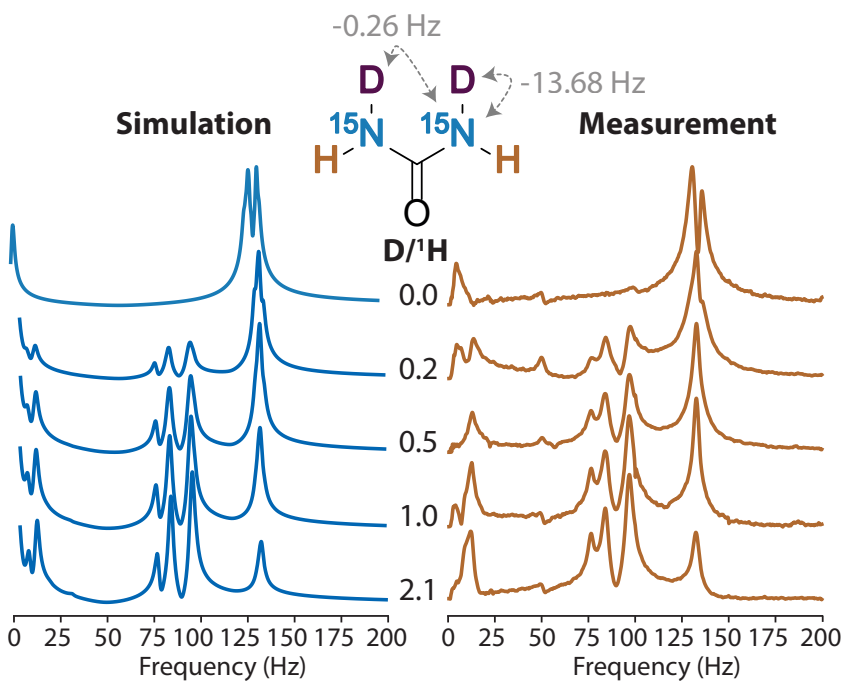

FIG. 3. Experimental and simulated zero-field $J$-spectra of $\left[{ }^{15} \mathrm{~N}_{2}\right]$-urea in aqueous solutions with various ${ }^{1} \mathrm{H} / \mathrm{D}$ ratios. $J$ coupling values used in simulations are shown with chemical structures of an exemplary ${ }^{1} \mathrm{H} / \mathrm{D}$ isotopologue of urea. All isotopologues' structures and corresponding simulated zerofield $J$-spectra are shown in SI.

mial distribution. Since a probability of an amide-proton site being occupied by deuterium depends on the fraction $p$ of deuterium in the solution, the molar fraction $x$ of each isotopologue is given by

$$
x=\left(\begin{array}{l}
n \\
k
\end{array}\right) p^{k}(1-p)^{n-k}
$$

where $n$ is the number of possible sites where $\mathrm{D}$ nuclei can reside and $k$ is the number of $\mathrm{D}$ nuclei that each isotopologue contains. Simulated spectra of all isotopologues are next summed after weighing each spectrum with an appropriate binomial coefficient. For the simulation, the D- ${ }^{15} \mathrm{~N}$ coupling constants are estimated using the ratio of appropriate gyromagnetic ratios of the nuclei and the $J_{\mathrm{NH}}$ coupling constant, $J_{\mathrm{ND}} \approx\left(\gamma_{D} / \gamma_{H}\right) J_{\mathrm{NH}}$, which neglects secondary isotope effects [33]. As a result, we obtained a good agreement of the experimental spectra of urea solutions with the various ratios of $\mathrm{D}_{2} \mathrm{O} / \mathrm{H}_{2} \mathrm{O}$ with their simulated counterparts (Fig. 3).

In the analysis of the urea ${ }^{1} \mathrm{H} / \mathrm{D}$ isotopologues $J$ spectra, two nitrogen atoms are treated as equivalent hence the isotopologues consist of three different spin subsystems: $-\mathrm{NH}_{2}-$ an $\mathrm{XA}_{2}$ spin system, -NHD - an (XA)B spin system, and $-\mathrm{ND}_{2}-$ an $\mathrm{XB}_{2}$ spin system. As shown in Fig. 4, the peaks arising from $-\mathrm{NH}_{2}$ and -NHD groups are predicted using the first-order perturbation theory. It should be also noted that signals from the $\mathrm{ND}_{2}$ group are not observed in the spectra. This results from the fact that amplitude of ZULF NMR signals is proportional to the square of the difference between gyromagnetic ratios of $J$-coupled nuclei [7], which in the considered case equals $\left(\gamma_{D}-\gamma_{15}\right)^{2} /\left(\gamma_{1} H-\gamma_{15}\right)^{2} \approx 0.0025$ (see SI for detailed energy-level analysis).

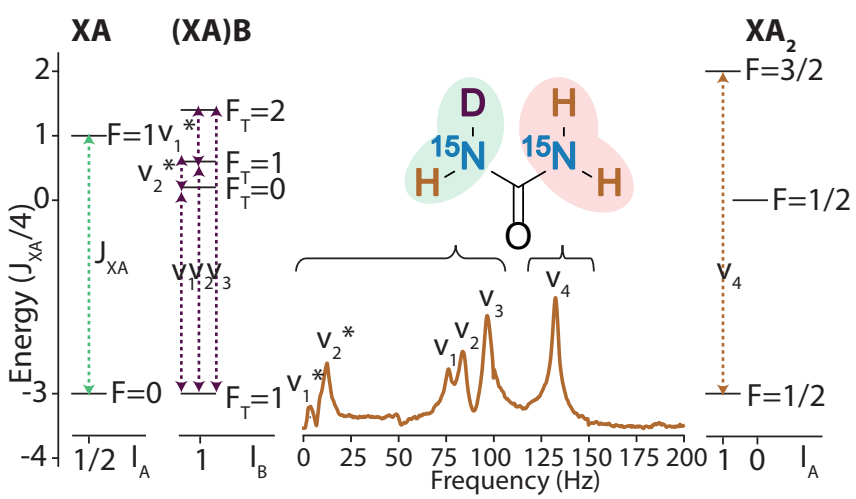

FIG. 4. a Schematic energy level structures for XA, (XA)B and $\mathrm{XA}_{2}$. High and low-frequency transitions in (XA)B spin system are denoted by $\mathrm{v}_{1-3}$ and $\mathrm{v}_{1-2}^{*}$, respectively. The transition in $\mathrm{XA}_{2}$ spin system is represented as $\mathrm{v}_{4}$ which corresponds to $3 / 2 J_{\mathrm{XA}}$. The manifolds are grouped by the quantum numbers $I_{A}$ and $I_{B}$ that denote the spin number of $\mathrm{A}$ nuclei and B nuclei, respectively. Each manifold is labelled by its total spin quantum number $F$ or $F_{T}$ (see SI for detailed energy level analysis) [5, 9]. Only a single sublevel in each manifold and a single transition at each frequency are shown for clarity. b Experimental spectra of $\left[{ }^{15} \mathrm{~N}_{2}\right]$-urea in the mixture of $\mathrm{D}_{2} \mathrm{O} / \mathrm{H}_{2} \mathrm{O}(1: 1)$. For all peaks in the spectrum, the corresponding transitions $\left(\mathrm{v}_{1-4}, \mathrm{v}_{1-2}^{*}\right)$ are determined by a first-order perturbation theory.

The observation of quadrupolar nuclei (spin $>1 / 2$ ) in zero-field NMR is challenging due to their additional electric influence on reorientation of nuclei that can cause fast relaxation [30]. Previous studies show that even though signals from the $J$-coupling to $\mathrm{D},{ }^{14} \mathrm{~N}$, and ${ }^{35 / 37} \mathrm{Cl} \mathrm{nu}-$ clei may not be directly visible in zero-field spectra, they may cause additional line-broadening [5, 34]. Conversely, in the study on zero-field NMR of quadrupolar nuclei, peaks originating from $J$-coupling interactions of ${ }^{1} \mathrm{H}-\mathrm{D}$ and ${ }^{1} \mathrm{H}^{14}{ }^{14} \mathrm{~N}$ are demonstrated in $J$-spectra of ammonium isotopologues [35]. Due to relatively small electric moment of D, and high local symmetry of ${ }^{14} \mathrm{~N}$-ammonium resulting in small nuclear quadrupolar interactions, the detection of $J$-coupling interactions of such nuclei in zerofield NMR is feasible [30]. Our results demonstrate that zero-field NMR is able to detect ${ }^{1} \mathrm{H} / \mathrm{D}$ isotopologues of urea molecules as well as provide information on ${ }^{1} \mathrm{H} / \mathrm{D}$ ratio in a solution through simulation of $J$-spectrum. We also show that $J$-spectra for the complex molecules with more than two heteronuclei can be interpreted clearly by analyzing the energy structure of each small spin subgroup separately.

To summarize, we investigated urea, one of the crucial biomolecules, under various solution conditions using ZULF NMR. We demonstrated that the compound can be readily detected in water by zero-field NMR, modifying spin topology via an chemical-exchange procedure. Our results can be extrapolated to other biomolecules with similar structures (e.g., amino acids) opening the technique for various biochemical research. We also re- 
ported that the $J$-spectra of complex molecules, such as urea isotopologues, can be clearly interpreted by identifying simple subgroups in the system and analysing their energy structures independently. All the experimental results are supported with simulations, supporting our theoretical interpretation. In turn, the work implies the ability of future in vivo/in vitro biochemical investigations of complex molecules. For example, such studies will be possible in biofluids (e.g., blood, urine, etc.) with a high concentration of water, which, in the conventional ${ }^{1} \mathrm{H}$ NMR, forms a challenge. Specifically, one of significant clinical analysis methods, the quantification of urea in urine and blood, will be a subject of our future research. In this context, however, it should be noted that in the presented study, we worked with highly concentrated (5-8 M), isotopically-enriched urea solutions. Even with such high concentrations, low thermal prepolarization, provided by the 1.8-T magnet, results in low amplitude of ZULF NMR signals. To overcome this limitation, zero-field NMR may be combined with hyperpolarization methods such as parahydrogen-induced polarization (PHIP), [36, 37], signal amplification by reversible exchange (SABRE), [38] dynamic nuclear polarization (DNP) [11], etc. As these methods are limited to a few suitable molecules, in our future experiments we plan to address the problem by the exchange-based polarization methods: SABRE-RELAY and PHIP-X that provide wide applicability for diverse biomolecules [31, 32].

\section{EXPERIMENTAL METHODS}

\section{A. Sample preparation}

All chemicals were purchased from Sigma-Aldrich and used without further purification. $\quad\left[{ }^{13} \mathrm{C},{ }^{15} \mathrm{~N}_{2}\right]$-urea (CAS\# 58069-83-3) and $\left[{ }^{15} \mathrm{~N}_{2}\right]$-urea (CAS\# 2067-80-3) solutions at various $\mathrm{pH}$ values were prepared in a $8-\mathrm{M}$ concentration by dissolving in prepared different concentrations of sodium hydroxide (CAS\# 1310-73-2) or hydrochloric acid (CAS\# 7647-01-0). The pH of each sample was measured at room temperature using a portable pH meter (Mettler Toledo Seven2Go) with a micro electrode (Mettler-Toledo InLab Pro-ISM). For the study of the ${ }^{1} \mathrm{H}-\mathrm{D}$ exchange in urea, 8-M $\left[{ }^{15} \mathrm{~N}_{2}\right]$-urea solutions were prepared by dissolving in distilled water, $\mathrm{D}_{2} \mathrm{O}$ (CAS\# 7789-20-0) and 25\%, 50\%, 75\% distilled water$\mathrm{D}_{2} \mathrm{O}$ (CAS\# 7789-20-0) mixtures. For preparation of urea solutions in DMSO, $\left[{ }^{13} \mathrm{C},{ }^{15} \mathrm{~N}_{2}\right]$-urea (CAS\# 5806983-3) and $\left[{ }^{15} \mathrm{~N}_{2}\right]$-urea were dissolved in DMSO (CAS\# 67-68-5) with a final concentration of 5.4 M. Each sample $(0.15 \mathrm{ml})$ was loaded into a standard 5 -mm NMR tube and then flame-sealed under vacuum $\left(<10^{-4} \mathrm{mbar}\right)$ following degassing by several freeze-pump-thaw cycles.

\section{B. Zero-field NMR experiment}

The NMR samples are thermally polarized for $20 \mathrm{~s}$ using a 1.8-T magnet placed above the magnetic shield, and mechanically shuttled into a zero-field detection region (inside the magnetic shield), where the magnetic field of the nuclear spins is measured using a home-built alkalivapor atomic magnetometer. During the transfer, taking roughly $300 \mathrm{~ms}$ (plus an additional 700-ms delay), a guiding field of roughly $10 \mu \mathrm{T}$ is applied by a solenoid coil wrapping the whole length of the shuttling path. When the sample reaches the detection area, the guiding field is turned off suddenly to generate an oscillating NMR signal [7]. Each zero-field NMR spectra is the result of averaging 2048 transients. The entire data processing is performed using Python. A comprehensive description of the experimental setup and a detailed explanation of data processing can be found in the previous work [5].

\section{Zero-field NMR simulations}

A high-performance spin simulation library Spintrum is employed to simulate zero-field NMR spectra through a numerical diagonalization of a density matrix describing the spin system $[3,39]$. The $J$-coupling values in the simulations are taken directly from the literature or estimated using the gyromagnetic ratios of nuclei (see discussion above). Simulations of chemical-exchange effects on the zero-field spectra of urea were obtained using an approach presented in Ref. [11]. Details of the calculations as well as a discussion of possible shortcomings of the used exchange model are discussed in SI.

\section{ACKNOWLEDGMENTS}

The authors acknowledge the support from the European Union's Horizon 2020 research and innovation program under the Marie Skłodowska-Curie grant agreement No. 766402. S.A., P.P., and S.P. acknowledge the support from the Talent Management mini-grant within the framework of the Anthropocene Priority Research Area (Project No. PSP U1U/P07/DO/14.24).

\section{Supporting Information}

Zero-field NMR signal amplitude of urea versus guiding field; frequencies of resonance lines in deuterated urea $J$-spectra; simulated spectra of deuterated urea isotopologues; details of chemical exchange simulations in zerofield. The experimental data will be available upon request. 


\section{Competing interests}

The authors declare no competing interests.

[1] J. W. Blanchard, D. Budker, and A. Trabesinger, Journal of Magnetic Resonance 323, 106886 (2021).

[2] M. C. D. Tayler, T. Theis, T. F. Sjolander, J. W. Blanchard, A. Kentner, S. Pustelny, A. Pines, and D. Budker, Review of Scientific Instruments 88, 091101 (2017).

[3] A. Wilzewski, S. Afach, J. W. Blanchard, and D. Budker, Journal of Magnetic Resonance 284, 66 (2017).

[4] J. W. Blanchard, M. P. Ledbetter, T. Theis, M. C. Butler, D. Budker, and A. Pines, Journal of the American Chemical Society 135, 3607 (2013).

[5] S. Alcicek, P. Put, V. Kontul, and S. Pustelny, The Journal of Physical Chemistry Letters 12, 787 (2021).

[6] P. Put, S. Pustelny, D. Budker, E. Druga, T. F. Sjolander, A. Pines, and D. A. Barskiy, Analytical Chemistry 93, 3226 (2021).

[7] J. W. Blanchard and D. Budker, eMagRes 5, 1395 (2016).

[8] T. Theis, J. W. Blanchard, M. C. Butler, M. P. Ledbetter, D. Budker, and A. Pines, Chemical Physics Letters 580, 160 (2013).

[9] M. C. Butler, M. P. Ledbetter, T. Theis, J. W. Blanchard, D. Budker, and A. Pines, The Journal of Chemical Physics 138, 184202 (2013).

[10] A. Bain, in Encyclopedia of Spectroscopy and Spectrometry (Third Edition), edited by J. C. Lindon, G. E. Tranter, and D. W. Koppenaal (Academic Press, Oxford, 2017) third edition ed., pp. 180-187.

[11] D. Barskiy, M. Tayler, I. Marco-Rius, J. Kurhanewicz, D. Vigneron, S. Cikrikci, A. Aydogdu, M. Reh, A. Pravdivtsev, J.-B. Hövener, J. Blanchard, W. Teng, D. Budker, and A. Pines, Nature Communications 10, 3002 (2019).

[12] D. Burueva, J. Eills, J. Blanchard, A. Garcon, R. PicazoFrutos, K. Kovtunov, I. Koptyug, and D. Budker, Angewandte Chemie International Edition 59, 17026 (2020).

[13] I. D. Weiner, W. E. Mitch, and J. M. Sands, Clinical Journal of the American Society of Nephrology 10, 1444 (2015).

[14] A. J. Taylor and P. Vadgama, Annals of Clinical Biochemistry 29, 245 (1992).

[15] L. Liu, H. Mo, S. Wei, and D. Raftery, Analyst 137, 595 (2012).

[16] C. Pundir, S. Jakhar, and V. Narwal, Biosensors and Bioelectronics 123, 36 (2019).

[17] Z. J. Wang, M. A. Ohliger, P. E. Z. Larson, J. W. Gordon, R. A. Bok, J. Slater, J. E. Villanueva-Meyer, C. P. Hess, J. Kurhanewicz, and D. B. Vigneron, Radiology 291, 273 (2019).

[18] P. M. Nielsen, E. S. Szocska Hansen, T. S. Nørlinger, R. Nørregaard, L. Bonde Bertelsen, H. Stødkilde Jørgensen, and C. Laustsen, Magnetic
Resonance in Medicine 76, 1524 (2016).

[19] A. Z. Lau, J. J. Miller, M. D. Robson, and D. J. Tyler, Magnetic Resonance in Medicine 75, 1474 (2016).

[20] P. Francis, S. Lewis, and K. Lim, TrAC Trends in Analytical Chemistry 21, 389 (2002).

[21] P. W. Kuchel, C. Naumann, B. E. Chapman, D. Shishmarev, P. Håkansson, G. Bacskay, and N. S. Hush, Journal of Magnetic Resonance 247, 72 (2014).

[22] O. Steinhof, E. J. Kibrik, G. Scherr, and H. Hasse, Magnetic Resonance in Chemistry 52, 138 (2014).

[23] S. Appelt, F. W. Häsing, U. Sieling, A. Gordji-Nejad, S. Glöggler, and B. Blümich, Phys. Rev. A 81, 023420 (2010).

[24] R. L. Vold, E. Daniel, and S. Chan, Journal of the American Chemical Society 92, 6771 (1970).

[25] P. Giraudeau, V. Silvestre, and S. Akoka, Metabolomics 11 (2015).

[26] J. Bell, W. A. Gillespie, and D. B. Taylor, Trans. Faraday Soc. 39, 137 (1943).

[27] I. Yavari and J. D. Roberts, Organic Magnetic Resonance 13, 68 (1980).

[28] C. L. Perrin, E. R. Johnston, C. P. Lollo, and P. A. Kobrin, Journal of the American Chemical Society 103, 4691 (1981).

[29] I. M. Klotz and D. L. Hunston, The Journal of Physical Chemistry 75, 2123 (1971).

[30] M. Levitt, Spin Dynamics: Basics of Nuclear Magnetic Resonance, Second Edition (John Wiley \& Sons, 2008).

[31] W. Iali, P. J. Rayner, and S. B. Duckett, Science Advances 4, 10.1126/sciadv.aao6250 (2018).

[32] K. Them, F. Ellermann, A. N. Pravdivtsev, O. G. Salnikov, I. V. Skovpin, I. V. Koptyug, R. Herges, and J.-B. Hövener, arXiv:2012.03626 [physics.chem-ph] (2020).

[33] E. Canet, D. Mammoli, P. Kadeřávek, P. Pelupessy, and G. Bodenhausen, Phys. Chem. Chem. Phys. 18, 10144 (2016).

[34] M. C. Tayler and L. F. Gladden, Journal of Magnetic Resonance 298, 101 (2019).

[35] D. Barskiy, J. Blanchard, M. Reh, T. Sjoelander, A. Pines, and D. Budker, arXiv:2011.05618 [physics.chem-ph] (2020).

[36] T. Theis, P. Ganssle, G. Kervern, S. Knappe, J. Kitching, M. P. Ledbetter, D. Budker, and A. Pines, Nature Physics 7, $571-575$ (2011).

[37] M. C. Butler, G. Kervern, T. Theis, M. P. Ledbetter, P. J. Ganssle, J. W. Blanchard, D. Budker, and A. Pines, Journal of Chemical Physics 138, 234201 (2013).

[38] T. Theis, M. P. Ledbetter, G. Kervern, J. W. Blanchard, P. J. Ganssle, M. C. Butler, H. D. Shin, D. Budker, and A. Pines, Journal of the American Chemical Society 134, 3987 (2012).

[39] S. Afach, Spintrum (2018). 